\title{
The Use of a Quadratic Form for the Determination of Non- negative Texture Functions
}

\author{
P. VAN HOUTTE \\ Department Metaalkunde, Katholieke Universiteit Leuven, de Croylaan 2, \\ B-3030 Leuven, Belgium
}

(Received January 11, 1983)

The classical analysis of measured pole figures of textured polycrystals by the series expansion method does not necessarily produce a non-negative texture function. The main reason for this is, that the method is unable to find the terms of odd rank $l$ of the series expansion.

A new method is proposed, which introduces the non-negativity condition into the series expansion method by the use of quadratic forms. The method is found to be successful when treating sharp textures, which have a considerable zero range in Euler space. The preliminary determination of this zero range by experimental methods is however not necessary.

\section{INTRODUCTION}

The well-known series expansion methods allows the calculation of the crystal orientation distribution function of a polycrystal from measured pole figures (Bunge, 1969).

The O.D.F. is written as follows

$$
f(g)=\sum_{l=0}^{\infty} \sum_{\mu=1}^{M(l)} \sum_{\nu=1}^{N(l)} C_{l}^{\mu \nu} \dot{T}_{l}^{\mu \nu}(g)
$$

The $C_{l}^{\mu v}$ coefficients are to be obtained from measured data such as pole figures.

An orientation distribution function cannot be negative because of 
its statistical nature. This property is unfortunately not built-in in the series expansion of Eq. 1. Many researchers who have used the series expansion method have observed negative values of their O.D.F.

There are three reasons for this:

i) Small errors in the measured pole figures.

ii) Truncation of the series expansion at some maximal value $L$ of $l$.

iii) Omission of the terms with odd values of $l$.

As was indeed first pointed out by Matthies (1979), the classical method for analysis of measured pole figures only produces the so-called "reduced O.D.F.", which corresponds to the terms of even rank $l$ :

$$
\tilde{f}(g)=\sum_{l=0(2)} \sum_{\mu} \sum_{v} C_{l}^{\mu \nu} \dot{T}_{l}^{\mu \nu}(g)
$$

The true O.D.F. is then given by:

$$
f(g)=\widetilde{f}(g)+\tilde{f}(g)
$$

where $\tilde{f}(g)$ corresponds to the terms of odd rank $l$.

The true O.D.F. is usually zero in a large part of Euler space ("zero range") when the texture is sharp. In such case, the reduced O.D.F. can have rather important negative peaks. False positive peaks ("ghost maxima") may appear as well. Moreover, it will usually underestimate the value of the true maxima.

Bunge and Esling (1979) have suggested that the non-negativity condition could be used in order to estimate the odd part $f(g)$ of the texture function. They have proposed a method which requires the preliminary determination of the zero range. Results have recently been presented (Bechler-Ferry, Bunge and Esling, 1981). The method tries to satisfy Eq. 3 in the zero range. It does not necessarily satisfy the non-negativity condition outside the predetermined zero range. Truncation effects might still cause minor negative values in the zero range.

Lücke, Pospiech, Virnich and Jura (1981) have proposed to model each particular O.D.F. by one or more Gaussian distributions. The reduced O.D.F. derived from the model must then fit the reduced O.D.F. obtained from measured pole figures. An estimation of $\widetilde{\tilde{f}}(g)$ can be calculated once a good model is found. The success of the method depends upon the effort spent in order to find a suitable model for each particular case.

The new method presented here automatically satisfies the non- 
negativity condition without requiring the preliminary knowledge of the zero range.

The basic idea is very simple: the O.D.F. is expressed in a quadratic form, and hence cannot be negative:

$$
f_{s}(g)=h_{s}^{2}(g) \geqslant 0
$$

An iterative method is used in order to find the function $h_{s}(g)$ which produces a satisfactory estimation $f_{s}(g)$ of the true O.D.F. $f(g)$.

All equations below are written for the case that $C_{l}^{\mu \nu}$ and $\dot{T}_{l}^{\mu v}$ are real (cubic-orthorombic symmetry). It is possible to adapt the equations to other cases.

\section{ERROR CRITERION}

Version (a) of the method will try to fit the reduced O.D.F. associated to the quadratic form with the reduced O.D.F. obtained from the measurements. The reduced O.D.F. can be obtained from several measured incomplete pole figures (Pospiech and Jura, 1974; Morris, 1975; Humbert and Bergmann, 1980; Van Houtte, 1980; Lian, Xu and Wang, 1981). In case complete pole figures are available, it is better to use version (b), which tries to fit the pole figures recalculated from the quadratic form directly with the measured pole figures.

Version (a) and (b) will each have their own error criterion.

Let $f_{s}(g)$ be an estimation of the true texture function $f(g) . f_{s}(g)$ can be written as a series expansion:

$$
f_{s}(g)=\sum_{l=0}^{L} \sum_{\nu=1}^{N(l)} \sum_{\mu=1}^{M(l)} C_{s_{l}}^{\mu \nu} \dot{T}_{l}^{\mu \nu}
$$

$l$ takes even and odd values.

The coefficients $C_{s_{1}}^{\mu v}$ can be found by numerical integration when the estimation $f_{s}(g)$ is known (Bunge, 1969).

\section{Version (a) of the method}

The reduced O.D.F. is known.

$$
f(g)=\sum_{l=0(2)}^{L} \sum_{v=1}^{N(l)} \sum_{\mu=1}^{M(l)} C_{l}^{\mu v} \dot{T}_{l}^{\mu v}(g)
$$


The following criterion is proposed for the "difference" between $f(g)$ and $\tilde{f}_{s}(g)$ :

$$
\Delta_{s}=\sum_{l=0(2)}^{L} \sum_{\nu=1}^{N(l)} \sum_{\mu=1}^{M(l)} \frac{1}{2 l+1}\left(C_{s_{l}}^{\mu \nu}-C_{l}^{\mu \nu}\right)^{2}
$$

to be minimized

\section{Version (b) of the method}

Let there be $n$ measured pole figures. The proposed criterion is given by

$$
\Delta_{s}=\sum_{i=1}^{n} w_{i} \sum_{l=0(2)}^{L} \sum_{v=1}^{N(l)}\left[F_{s_{l}}^{v}\left(h_{i}\right)-F_{l}^{v}\left(h_{i}\right)\right]^{2}
$$

= Minimum

$F_{l}^{v}\left(h_{i}\right)$ are the $F$-coefficients (Bunge, 1969) of the observed pole figure $h_{i}, w_{i}$ is the corresponding weighing factor.

$$
F_{s_{l}}^{v}\left(h_{i}\right)=\frac{4 \pi}{2 l+1} \sum_{\mu=1}^{M(l)} C_{s_{l}}^{\mu v} \stackrel{\dot{m}}{k_{l}^{\mu}}\left(h_{i}\right)
$$

is obtained from the $C_{s_{1}}^{\mu \nu}$ coefficients.

Both criteria define a sum of squares $\Delta_{s}$ to be minimized. The method for adjusting the function $h_{s}(g)$ (Eq. (4)) has the character of a least squares minimization method.

\section{ELABORATION OF THE QUADRATIC FORM}

Equation (4) gives an estimation of the true texture function by a quadratic form $h_{s}^{2}(g)$. We will use a form for $h_{s}^{2}(g)$ by which the estimation $f_{s}(g)$ is automatically normalized.

$$
f_{s}(g)=h_{s}^{2}(g)=\frac{h^{2}(g)}{I}
$$

with

$$
h(g)=\sum_{l=0}^{L} \sum_{\nu=1}^{N(l)} \sum_{\mu=1}^{M(l)} H_{l}^{\mu \nu} \dot{T}_{l}^{\mu \nu}(g)
$$

and

$$
I=\oint h^{2}(g) d g=\sum_{l=0}^{L} \sum_{v=1}^{N(l)} \sum_{\mu=1}^{M(l)} \frac{\left(H_{l}^{\mu \nu}\right)^{2}}{2 l+1}
$$


The function $f_{s}(g)$ is always normalized

$$
\oint f_{s}(g) d g=1
$$

because of (9) and (11)

It will now be attempted to find the coefficients $H_{l}^{\mu \nu}$ by a least squares method. To that purpose, the partial derivatives

$$
\frac{\partial \Delta_{s}}{\partial H_{l}^{\mu \nu}}
$$

must be calculated ( $\Delta_{s}$ from Eq. (6) or Eq. (7)).

Useful quantities are (see Eq. (5)):

$$
\begin{aligned}
& C_{s_{l}}^{\mu \nu}=(2 l+1) \oint f_{s}(g) \dot{T}_{l}^{\mu \nu} d g=\frac{2 l+1}{I} \oint \dot{T}_{l}^{\mu \nu} h^{2}(g) d g \\
& \frac{\partial C_{s_{l}}^{\mu \nu}}{\partial H_{l^{\prime}}^{\mu^{\prime} \nu^{\prime}}}=\frac{2}{I}\left[(2 l+1) \oint h(g) \dot{\tilde{T}}_{l}^{\mu \nu}(g) \dot{\tilde{T}}_{l^{\prime}}^{\mu^{\prime} v^{\prime}} d g-\frac{2 C_{s_{l}}^{\mu \nu} H_{l^{\prime}}^{\mu^{\prime} v^{\prime}}}{\left(2 l^{\prime}+1\right)}\right]
\end{aligned}
$$

\section{Version (a)}

$\Delta_{s}$ is defined by Eq. (6).

$$
\frac{\partial \Delta_{s}}{\partial H_{l^{\prime}}^{\mu^{\prime} \nu^{\prime}}}=2 \sum_{l=0(2)}^{L} \sum_{\mu} \sum_{\nu} \frac{1}{2 l+1}\left(C_{s_{l}}^{\mu \nu}-C_{l}^{\mu \nu}\right) \frac{\partial C_{s_{l}}^{\mu \nu}}{\partial H_{l^{\prime}}^{\mu^{\prime} \nu^{\prime}}}
$$

We define

$$
K_{s_{l}}^{\mu \nu}=C_{s_{l}}^{\mu \nu}-C_{l}^{\mu \nu}
$$

and

$$
\tilde{k}_{s}(g)=\sum_{l=0(2)}^{L} \sum_{\mu} \sum_{v} K_{s_{l}}^{\mu \nu} \dot{T}_{l}^{\mu \nu}
$$

Equation (14) now becomes:

$$
\frac{\partial \Delta_{s}}{\partial H_{l^{\prime}}^{\mu^{\prime} v^{\prime}}}=2 \sum_{l=0(2)} \sum_{\mu} \sum_{v} \frac{K_{s_{l}}^{\mu \nu}}{2 l+1} \frac{\partial C_{s_{l}}^{\mu v}}{\partial H_{l^{\prime}}^{\mu^{\prime} v^{\prime}}}
$$

\section{Version (b)}

$\Delta_{s}$ is defined by Eq. (7)

$$
\frac{\partial \Delta_{s}}{\partial H_{l^{\prime}}^{\mu^{\prime} v^{\prime}}}=2 \sum_{i} w_{i} \sum_{l=0(2)}^{L} \sum_{v}\left[F_{s_{l}}^{v}\left(h_{i}\right)-F_{l}^{v}\left(h_{i}\right)\right] \frac{\partial F_{s_{l}}^{v}}{\partial H_{l^{\prime}}^{\mu^{\prime} v^{\prime}}}
$$


From Eq. (8), it is seen that

$$
\frac{\partial F_{s_{l}}^{v}}{\partial H_{l^{\prime}}^{\mu^{\prime} v^{\prime}}}=\frac{4 \pi}{2 l+1} \sum_{\mu} \dot{\dot{k}_{l}^{\mu}}\left(h_{i}\right) \frac{\partial C_{s_{l}}^{\mu \nu}}{\partial H_{l^{\prime}}^{\mu^{\prime} v^{\prime}}}
$$

Hence

$$
\frac{\partial \Delta_{s}}{\partial H_{l^{\prime}}^{\mu^{\prime} v^{\prime}}}=\frac{8 \pi}{2 l+1} \sum_{l=0(2)}^{L} \sum_{\mu} \sum_{v}\left\{\sum_{i} w_{i}\left[F_{s_{l}}^{v}\left(h_{i}\right)-F_{l}^{v}\left(h_{i}\right)\right] \dot{k_{l}^{\mu}}\left(h_{i}\right)\right\} \frac{\partial C_{l}^{\mu \nu}}{\partial H_{l^{\prime}}^{\mu^{\prime} v^{\prime}}}
$$

We now define

$$
K_{s_{l}}^{\mu v}=4 \pi \sum_{i} w_{i}\left[F_{s_{l}}^{v}\left(h_{i}\right)-F_{l}^{v}\left(h_{i}\right)\right] \dot{k_{l}^{\mu}}\left(h_{i}\right)
$$

and

$$
\tilde{k_{s}}(g)=\sum_{l=0(2)} \sum_{\mu} \sum_{v} K_{s_{l}}^{\mu v} \dot{T}_{l}^{\mu v}(g)
$$

From (20) and (21), it follows that

$$
\frac{\partial \Delta_{s}}{\partial H_{l^{\prime}}^{\mu^{\prime} v^{\prime}}}=2 \sum_{l=0(2)}^{L} \sum_{\mu} \sum_{v} \frac{K_{s_{l}}^{\mu \nu}}{2 l+1} \frac{\partial C_{s_{l}}^{\mu v}}{\partial H_{l^{\prime} v^{\prime}}^{\mu^{\prime}}}
$$

It is seen that Eq. (23) is formally identical to Eq. (17). From here on, the same equations can be used for version (a) and (b).

From Eq. (13) and Eq. (17) or (23) it follows that

$$
\begin{aligned}
\frac{\partial \Delta_{s}}{\partial H_{l^{\prime}}^{\mu^{\prime} v^{\prime}}}=\frac{4}{I}\left\{\oint\left[\sum_{l=0(2)}^{L} \sum_{\mu} \sum_{v} K_{s}^{\mu \nu} \dot{T}_{l}^{\mu v}(g)\right] \dot{T}_{l^{\prime}{ }^{\prime} v^{\prime}}(g) h(g) d g\right. \\
\left.-\frac{H_{l^{\prime}}^{\mu^{\prime} v^{\prime}}}{2 l^{\prime}+1}\left[\sum_{l=0(2)}^{L} \sum_{\mu} \sum_{v} \frac{K_{s_{l}}^{\mu \nu} C_{s_{l}}^{\mu \nu}}{2 l+1}\right]\right\}
\end{aligned}
$$

Let

$$
Q=\sum_{l=0(2)}^{L} \sum_{\mu} \sum_{v} \frac{K_{s_{l}}^{\mu v} C_{s_{l}}^{\mu v}}{2 l+1}
$$

Equation (24) now becomes (regarding Eq. (16) or (22))

$$
\frac{\partial \Delta_{s}}{\partial H_{l^{\prime}}^{\mu^{\prime} v^{\prime}}}=\frac{4}{I}\left[\oint \tilde{k}_{s}(g) h(g) \dot{\tilde{T}}_{l^{\prime} \nu^{\prime} v^{\prime}}(g) d g-\frac{Q H_{l^{\prime}}^{\mu^{\prime} v^{\prime}}}{2 l^{\prime}+1}\right]
$$

The evaluation of this expression is quite feasible by numerical integration. In the case of cubic crystal symmetry and orthorombic specimen symmetry, and for $L=22$, there are 185 different $H_{l}^{\mu \nu}$ coefficients. Assume that these are known at a certain iteration step. 
The following procedure will now be adopted in order to find a better estimation for $H_{l}^{\mu \nu}$.

First, the function $h(g)$ itself (Eq. (10)) will be computed at a grid pattern in Euler space. A mesh length of $3^{\circ}$ was used in the present work.

Equation (9) then allows the calculation of $f_{s}(g)$ for the points of the grid pattern.

The coefficients $C_{s_{l}}^{\mu v}$ of Eq. (5) are found by numerical integration:

$$
C_{s_{l}}^{\mu v}=(2 l+1) \oint \dot{T}_{l}^{\mu \nu}(g) f_{s}(g) d g
$$

It is now possible to calculate the $K_{s_{l}}^{\mu v}$ coefficients (Eq. (15) or Eq. (21) combined with Eq. $(8)) . \tilde{k_{s}}(g)$ is computed on the grid pattern using Eq. (16) or (22).

The 185 partial derivatives $\frac{\partial \Delta_{s}}{\partial H_{l^{\prime} v^{\prime}}^{\mu^{\prime}}}$ can be evaluated by numerical integration (Eq. (26)).

This finally produces a better value for $H_{l}^{\mu \nu}$ by the steepest descent method:

$$
\left(H_{l}^{\mu \nu}\right)_{\text {new }}=H_{l}^{\mu \nu}+\alpha \frac{\partial \Delta_{s}}{\partial H_{l}^{\mu \nu}}
$$

( $\alpha$ must be negative).

It is strongly advised to select a value for $\alpha$ which minimizes the new value of $\Delta_{s}$. This can be done by methods which require no additional computer time (see Appendix I).

This procedure should be repeated until it is seen that no significant decrease of $\Delta_{s}$ is obtained any more. A minimum for $\Delta_{s}$ as a function of the $H_{l}^{\mu v}$ is found by then.

At the present state of the analysis, it cannot be decided whether this minimum is a local minimum or "the" minimum.

Experience gained with case studies shows, that the results usually are very satisfactory.

The iteration scheme described above requires a first estimation of the $H_{l}^{\mu v}$-coefficients. Appendix II explains how this first estimation can be obtained.

The end result of the iteration is a set of $H_{l}^{\mu \nu}$-coefficients which can produce a non-negative O.D.F. (Eqs (9-11)). These coefficients can easily be normalized: 


$$
\left(H_{l}^{\mu \nu}\right)_{\text {normalized }}=\frac{H_{l}^{\mu \nu}}{\sqrt{I}}
$$

$I$ is given by Eq. (11). After normalization, the non-negative O.D.F. can be written as:

$$
f_{s}(g)=\left(\sum_{l=0}^{L} \sum_{\mu=1}^{M(l)} \sum_{\nu=1}^{N(l)} H_{l}^{\mu \nu} \dot{\dot{T}}_{l}^{\mu \nu}\right)^{2}
$$

This expression is very convenient for the calculation of $f_{s}(g)$ on sections through Euler space. A small intervention is enough to adopt existing O.D.F. computer programs to this formula.

Equation (12) can be used in order to produce the classical $C$ coefficients of $f_{s}$, as well for even as for odd values of $l$.

\section{CASE STUDIES}

The method was first tried out on a hypothetical texture consisting of

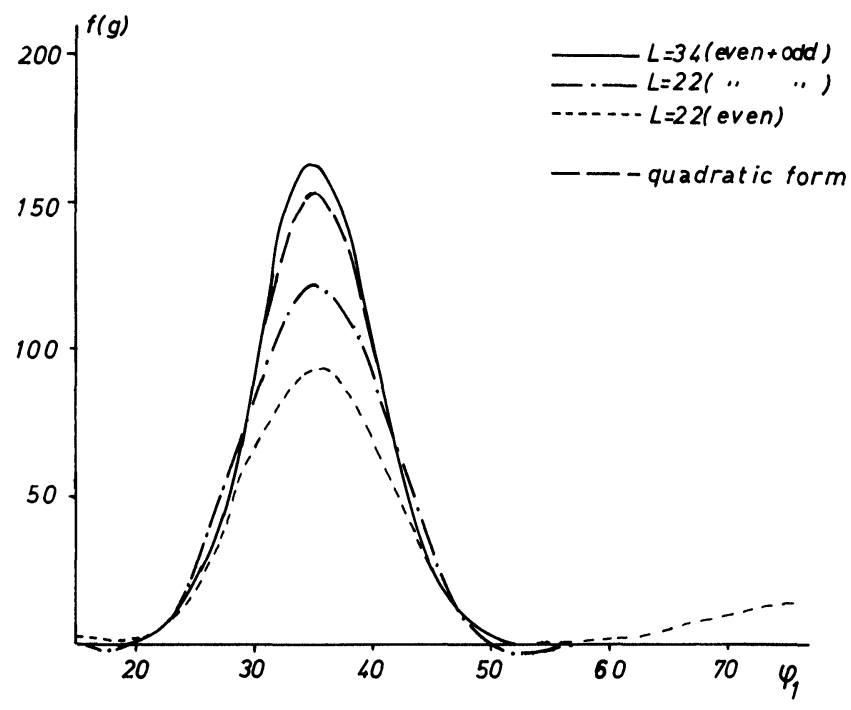

FIGURE 1 O.D.F. consisting of a Gaussian distribution $\left(\phi_{0}=7^{\circ}\right)$ around the brass orientation $\left(\phi_{2}=0^{\circ}, \Phi=45^{\circ}, \phi_{1}=35.26^{\circ}\right)$. Several approximations by series expansions are shown including a quadratic form obtained after 2 iteration steps.

$\phi_{2}=0^{\circ}, \Phi=45^{\circ}$ section plane. 
a Gaussian distribution (located at the brass-type texture) in the cubicorthorombic symmetry class. The centre of the Gaussian distribution was at $\phi_{1}=35.26^{\circ}, \phi_{2}=0^{\circ}, \phi=45^{\circ}$. The spread of the distribution $\left(\phi_{0}\right.$ in Eq. (6.45) used by Bunge (1969)) is $7^{\circ}$.

The $C_{l}^{\mu \nu}$ coefficients of this texture are known beforehand both for even and odd $l$. The even coefficients for $l$ up to 22 were used as input data for the test. Version (a) of the method was used.

The resulting quadratic form obtained after 2 iteration steps produced an O.D.F. without traces of ghost maxima. Figure 1 shows a $\phi_{2}=0$ and $\phi=0$ section through Euler space in which several functions are plotted:

- the true O.D.F. which in this plot cannot be distinguished from the classical series expansion for $l$ up to 34 (even and odd terms)

- the quadratic form $f_{s}(g)(L=22)$

- the classical series expansion for $l$ up to 22 (even and odd terms)

- the classical series expansion for $l$ up to 22 (even terms only)

It is seen that the quadratic form $f_{s}(g)$ obtained for $L=22$ is a far better approximation of the true O.D.F. than the classical series expan-

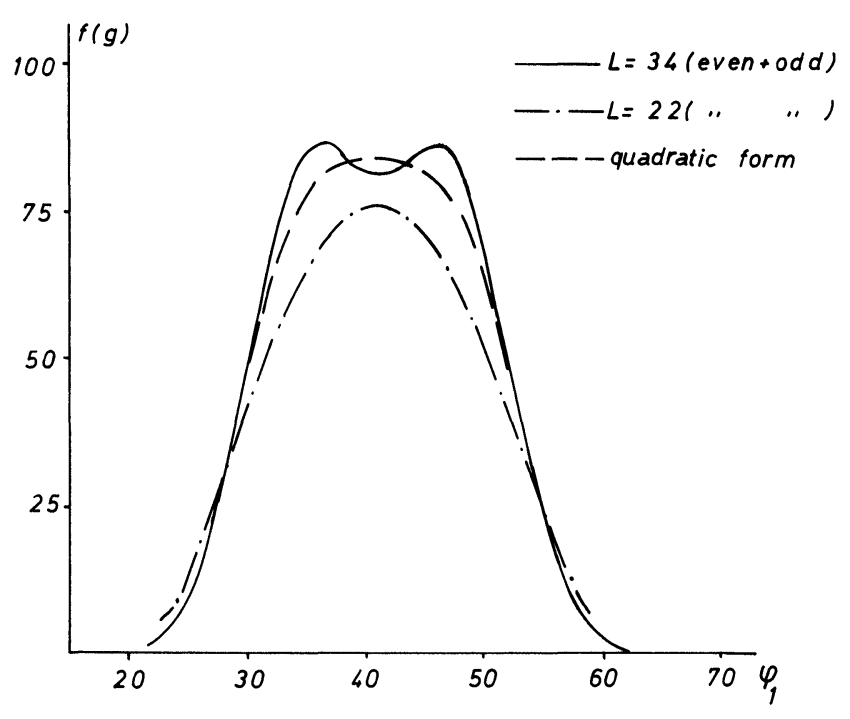

FIGURE 2 O.D.F. consisting of two Gaussian distributions $\left(\phi_{0}=7^{\circ}\right)$ of equal weight $\left(\phi_{2}=0^{\circ}, \Phi=45^{\circ}, \phi_{1}=35.26^{\circ}\right.$ and $\left.47^{\circ}\right)$. Several approximations by series expansions are shown, including a quadratic form obtained after 3 iteration steps. $\phi_{2}=0^{\circ}, \Phi=45^{\circ}$ section plane. 


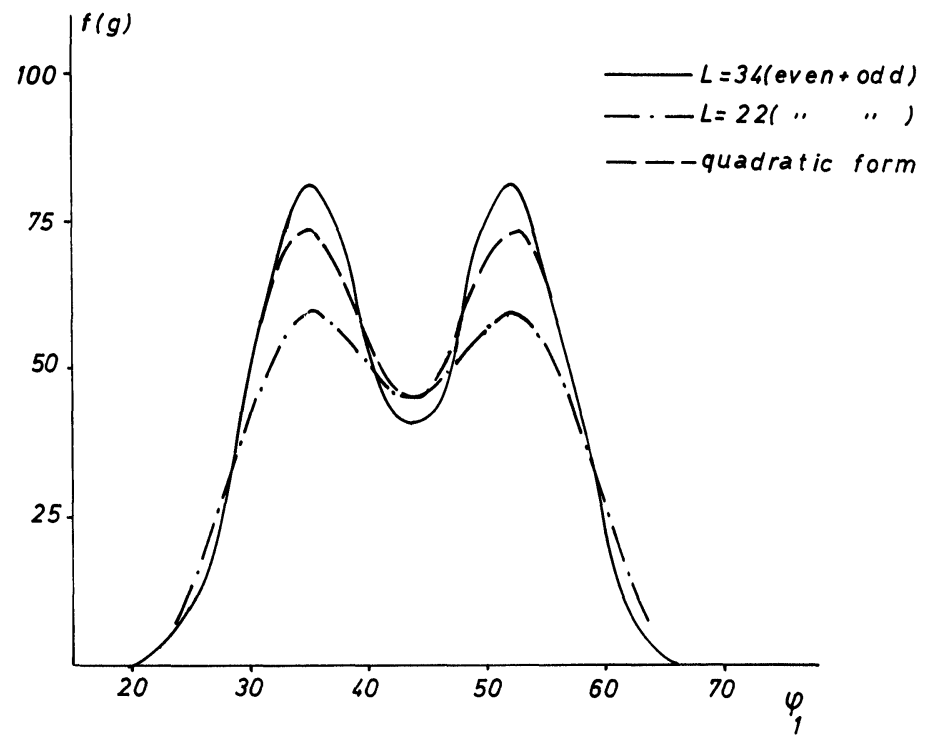

FIGURE 3 O.D.F. consisting of two Gaussian distributions $\left(\phi_{0}=7^{\circ}\right)$ of equal weight $\left(\phi_{2}=0^{\circ}, \Phi=45^{\circ}, \phi_{1}=35.26^{\circ}\right.$ and $\left.52^{\circ}\right)$. Several approximations by series expansions are shown, including a quadratic form obtained after 3 iteration steps. $\phi_{2}=0^{\circ}, \Phi=45^{\circ}$ section plane.

sion $L=22$ with both even and odd terms. Tests on more complex (but still sharp) textures have shown that this is a general tendency, but that the power of resolution of the method is nevertheless poorer than that of a classical series expansion with $L=34$ which would also include correct odd terms (Figures 2-3).

A subsequent test has been carried out on a complex texture of which both even and odd terms were known beforehand. This texture is a copper-type rolling texture simulated by the lath-version of the relaxed Taylor theory, as reported earlier (Van Houtte, 1981). The result of such a Taylor simulation is a set of 294 discrete crystallite orientations, each with its own weight. Such a texture is normally transformed into a continuous texture function $f(g)$ by putting a Gaussian distribution upon each of the 294 orientations.

The superposition of these 294 Gaussian distributions then produces the continuous texture function.

Both the even and odd $C_{l}^{\mu \nu}$ coefficients can easily be calculated for such textures. In Figure 5 given by Van Houtte (1981), Gaussian dis- 


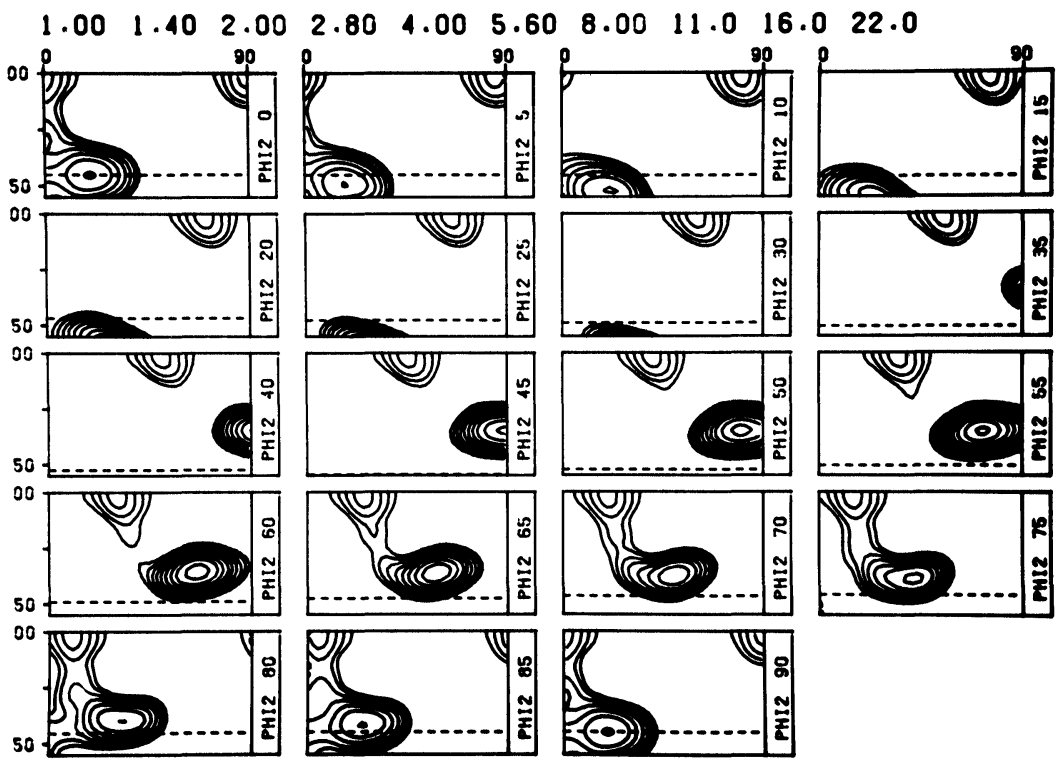

FIGURE $4 f(g)$ consists of 294 Gaussian distributions $\left(\phi_{0}=7^{\circ}\right)$ with each their own weight. The texture is a FCC rolling texture simulated by the relaxed Taylor theory (lath model, see Van Houtte, 1981). $L=34$, even and odd terms.

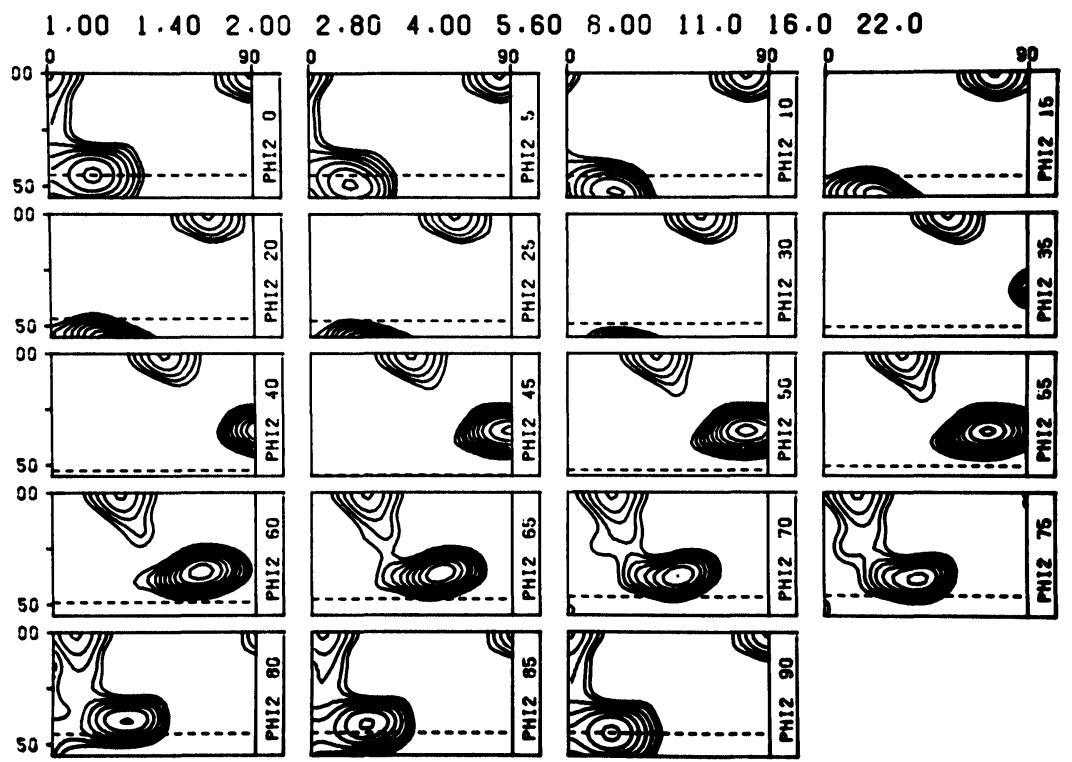

FIGURE 5 The quadratic form obtained after 3 iteration steps. The even $C$-coefficients ( $l$ up to 22 ) of the texture of Figure 4 were used as input data. 


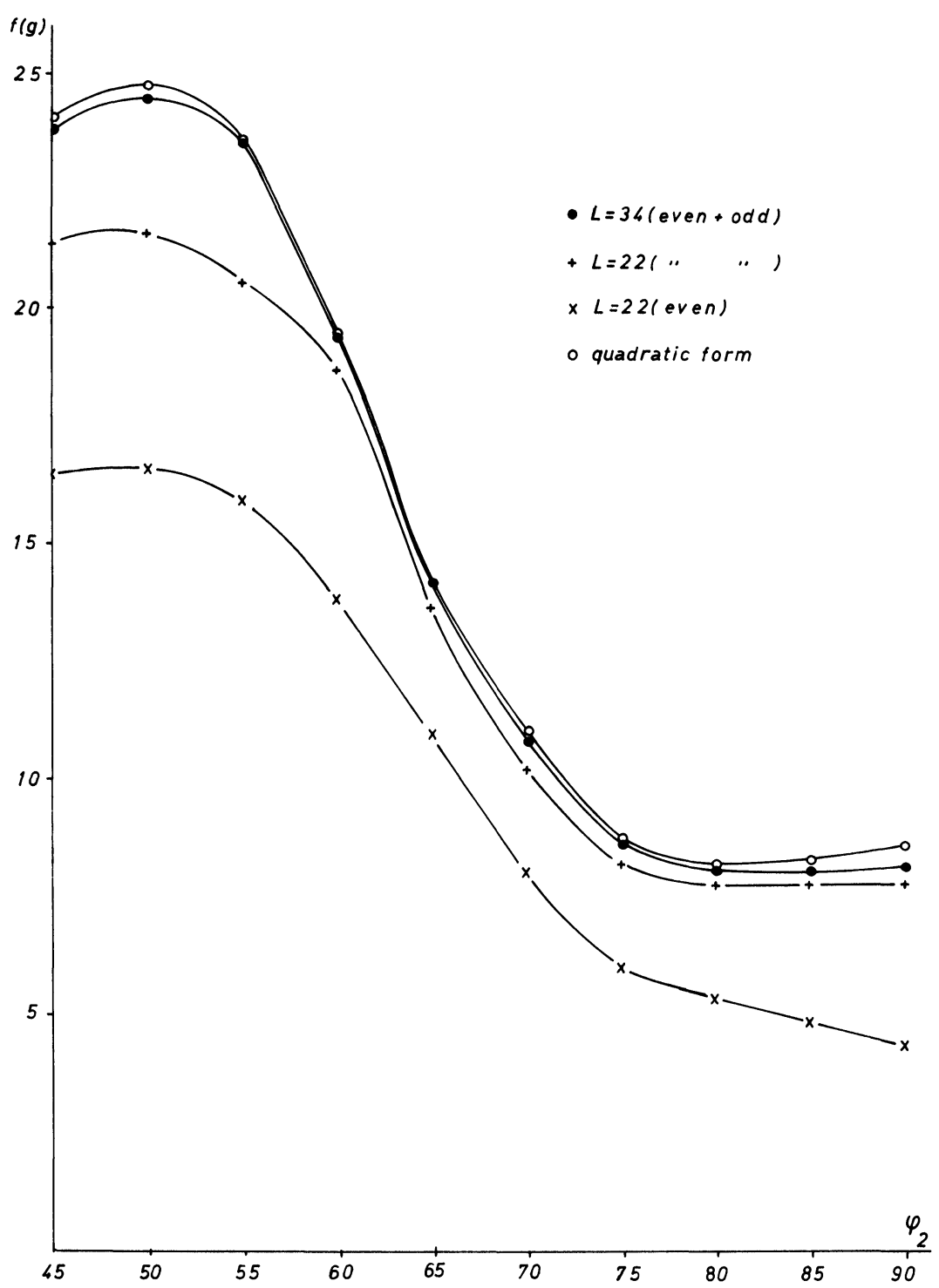

FIGURE 6 Variation of the approximation of $f(g)$ along the skeleton lines of Figures 4-5. The maximum values of the functions in sections of constant $\phi_{2}$ are given. 
tributions with a spread $\Phi_{0}=5^{\circ}$ were used. The series expansion was limited to $L=22$. Figure 4 of the present paper shows the result of the same Taylor simulation (lath model) but with $\Phi_{0}=7^{\circ}$ and $L=34$.

Of this texture, the even $C_{l}^{\mu \nu}$ coefficients for $l$ up to 22 were used as input data for a test of version (a) of the quadratic form method. After 3 iterations, the result of Figure 5 was found. Figure 6 shows the values of the texture functions of Figures 4-5 along the skeleton line. The results of the classical series expansion for $L=22$ (even + odd and even only) are also shown. It is seen that the quadratic form comes very close to the values obtained by the classical series expansion for $L=34$, the latter being undistinguishable from the correct value of the O.D.F. $\dagger$

It can be seen in Figures 4 and 5 that this excellent fitting in the vicinity of the maximum of the texture function is not always reached in the parts of Euler space where the function is weaker. There is however no trace left of ghost maxima.

The method has finally be applied on several textures obtained from incomplete pole figures measured by X-ray diffraction. The method made the ghost maxima disappear and enhanced the value of the principal maxima, especially in the case of sharp textures. One example will be given here: the deformation texture of a technically-pure copper specimen after $95 \%$ cold rolling. The texture has already been reported elsewhere (Van Houtte, 1981). Four incomplete pole figures have been analyzed producing the $C_{l}^{\mu \nu}$ coefficients of even rank $l$ up to 22 .

Figure 7 gives the reduced O.D.F. which contains ghost maxima. Its highest value is 16.33 . Figure 8 shows the quadratic form obtained after 6 iteration steps. The highest value is 26.74 .

It is interesting to note, that the pole figures recalculated from the quadratic form showed no excessive negative oscillations in their nonmeasured parts.

One iteration step of the quadratic form method with $L=22$ requires approximately the same amount of computer time as the usual calculation of the sections through Euler space of an O.D.F. by the series expansion method for $L=34$ (making extensive use of precalculated function libraries). In the case of very simple textures (Figure 1), as few iteration steps as 2 are sufficient; 4 steps are recommended for more complex textures.

$\dagger$ Gaussian distributions with $\phi_{0}=7^{\circ}$ can nearly exactly be fitted by a classical series expansion with $L=34$. 


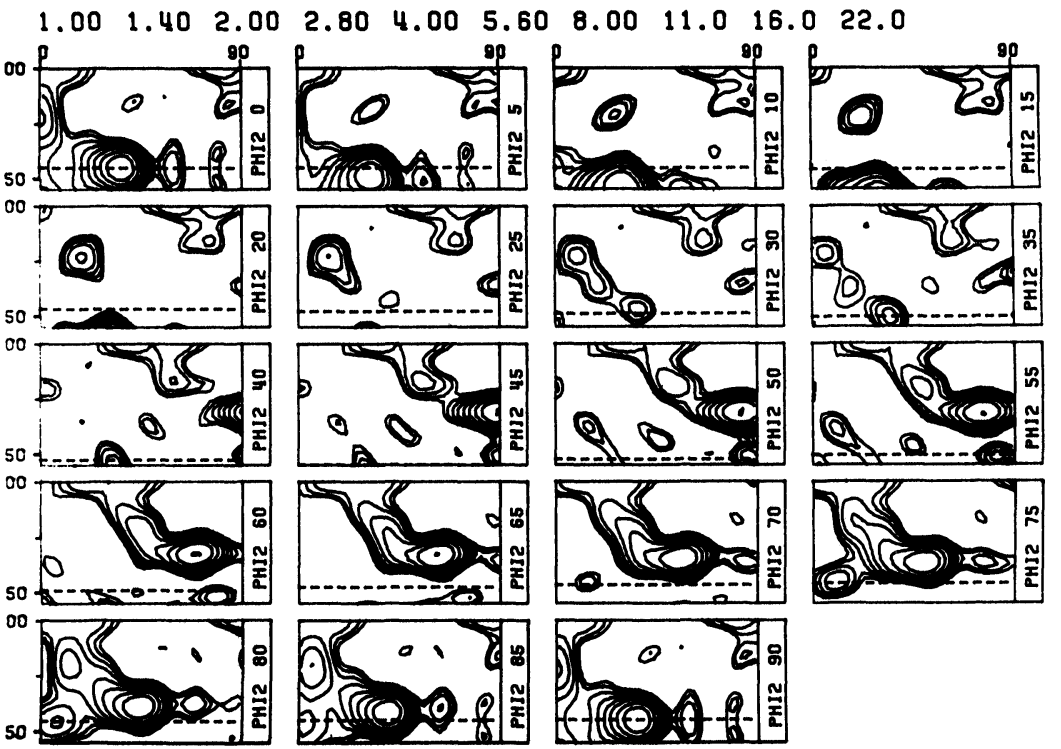

FIGURE 7 Reduced O.D.F. $(L=22)$ of a cold rolled copper specimen $(95 \%$ reduction).
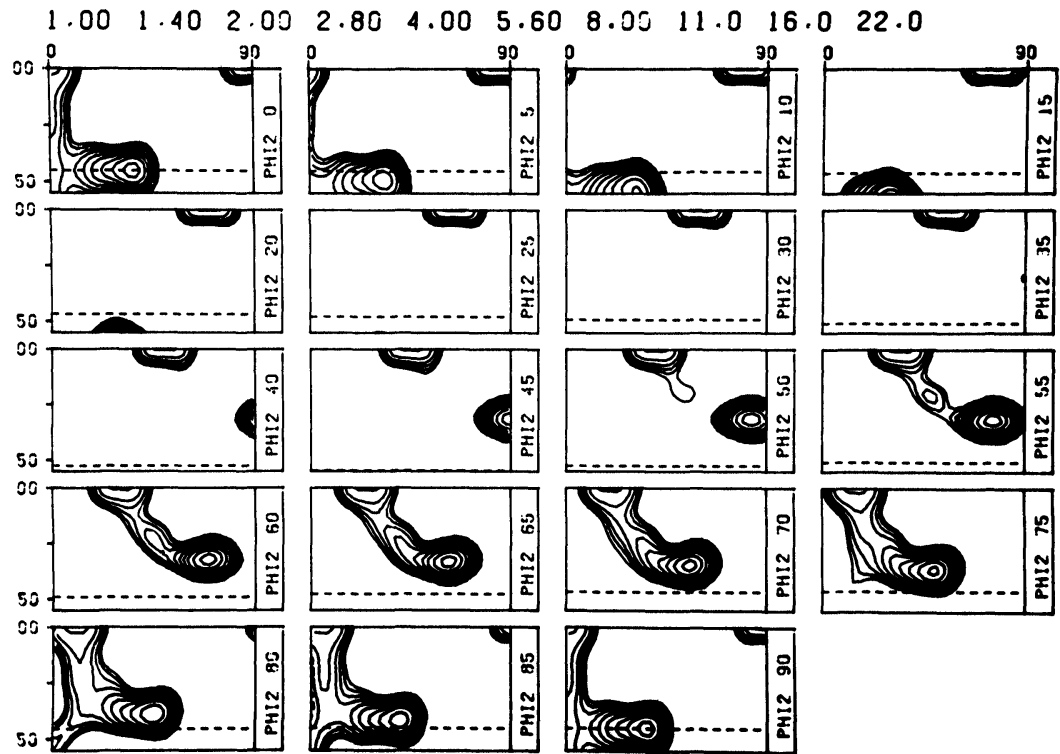

FIGURE 8 The quadratic form obtained from the reduced O.D.F. of Figure 7 (6 iteration steps). 


\section{DISCUSSION AND CONCLUSION}

It can be concluded from the nature of this method, that it can only provide information about the odd part of the texture function if the true O.D.F. has a considerable zero range.

It would be senseless to apply the present method upon a weak texture of which the reduced O.D.F. itself would have no negative values. According to Esling and Bunge (1981), this is a common feature of all methods which try to find the odd part of an O.D.F. by using the non-negativity condition.

Some textures which do not have a zero range have an isotropical background. In that case, the present method can also be used, provided this isotropical component is known. It could simply be subtracted from the texture, thus producing a new texture which has a zero range and upon which the non-negativity conditions can be applied.

Although the present method requires the existence of a zero range, its preliminar determination is not needed. It is capable of finding the zero range itself in the case of sufficiently sharp textures for which the variation range of $f(g)$ is strongly restricted by the positivity condition (Esling and Bunge, 1981).

Another advantage of the method is that the quadratic form gives a far better estimation of peak heights of sharp textures than the classical series expansion (even + odd terms) does at the same degree of expansion $(L=22)$. This result is nevertheless not so good as a classical series expansion (even + odd terms) up to $l=34$. The problem there is to find the odd $C_{l}^{\mu \nu}$ coefficients up to $l=33$. So far, only a method which models an O.D.F. by a superposition of various Gaussian distributions (Lücke et al., 1981) has been able to produce odd $C_{l}^{\mu \nu}$ coefficients up to such a high rank $l$. Such a method has the disadvantage that its success strongly depends upon good modelling of a particular O.D.F., i.e., it is not an automatic method.

The present method seems to be less accurate in the weaker regions of the O.D.F. than in the regions of high intensity. This problem might be related to the quadratic nature of the error criterion used.

At the present time, no computer programmes have been written for version (b) of the method which tries to fit complete pole figures directly. It can be expected on theoretical grounds that this version might even work if less than $M(L)$ pole figures have been measured (Bunge (1969), Figure 4.3), since the preliminar knowledge of the even $C_{l}^{\mu \nu}$ coefficients is not required in this case. For this reason, this version of the method 
might be very promising for the analysis of textures of non-cubic materials, where normally much more pole figures are required than in the case for cubic metals.

\section{References}

Bechler-Ferry, E., Bunge, H. J. and Esling, C. Proc. Sixth International Conference an Textures of Materials, pp. 1359-1369. Tokyo: The Iron and Steel Institute of Japan (1981).

Bunge, H. J. Mathematische Methoden der Texturanalyse, Berlin(DDR): Akademie-Verlag (1969).

Bunge, H. J. and Esling, C. Journal de Physique. 40, L627-L628 (1979).

Esling, C. and Bunge, H. J. Proc. Sixth International Conference on Textures of Materials, pp. 1426-1435. Tokyo: The Iron and Steel Institute of Japan (1981).

Humbert, M. and Bergmann, H. W. J. Appl. Cryst. 13, 500-504 (1980).

Liang, Z., Xu, J. and Wang, F. Proc. Sixth International Conference on Textures of Materials, pp. 1259-1264. Tokyo: The Iron and Steel Institute of Japan (1981).

Lücke, K., Pospiech, J., Virnich, K. H. and Jura, J. Acta Metall. 29, 167-185 (1981).

Matthies, S. Phys. Stat. Sol. (b) 92, K135-K138 (1979).

Morris, P. R. Adv. X-ray Anal. 18, 515-534 (1975).

Pospiech, J. and Jura, J. Z. Metallkde 27, 324-330 (1974).

Van Houtte, P. Mat. Sci. Eng. 43, 7-11 (1980).

Van Houtte, P. Proc. Sixth International Conference on Textures of Materials, pp. 428-437. Tokyo: The Iron and Steel Institute of Japan (1981).

\section{APPENDIX 1: OPTIMIZATION OF $\alpha$}

The method basically is a non-linear optimization method with quite a lot of unknown $H_{l}^{\mu \nu}$. Only the steepest descent method seems feasible, since most advanced optimization methods require the evaluation of the partial derivations of second order, which would cost too much computer time here.

The steepest descent method provides Eq. (28) as a means to find a better estimation of the variables:

$$
\left(H_{l}^{\mu v}\right)_{\text {new }}=H_{l}^{\mu v}+\alpha \frac{\partial \Delta_{s}}{\partial H_{l}^{\mu v}}
$$

The only thing known about $\alpha$ is that it must be negative. Unadequate choices of $\alpha$ would easily lead to unfruitful new estimates of the error criterion $\Delta_{s}$.

Therefore, a method has been developed to calculate the new error criterion $\Delta_{s+1}$ as a function of $\alpha$ and this without much computer cost.

The first step is to apply the normalization of $H_{l}^{\mu \nu}$ given by Eq. (29) 
on all intermediary $H_{l}^{\mu \nu}$ coefficients. This means that from here on, Eq. (30) is always valid. Let $f_{s}(g)$ be the quadratic form associated with $H_{l}^{\mu \nu}$, while $f_{s+1}(g)$ is associated with $\left(H_{l}^{\mu \nu}\right)_{\text {new }}$. We define now:

$$
h^{\prime}(g)=\sum_{l=0}^{L} \sum_{\mu=0}^{M(l)} \sum_{\nu=0}^{N(l)} \frac{\partial \Delta_{s}}{\partial H_{l}^{\mu \nu}} \dot{T}_{l}^{\mu \nu}(g)
$$

It is then seen from Eq. (28) and Eq. (29) that

$$
\begin{aligned}
f_{s+1}(g) & =\frac{\left[h(g)+\alpha h^{\prime}(g)\right]^{2}}{\oint\left[h(g)+\alpha h^{\prime}(g)\right]^{2} d g} \\
& =\frac{h^{2}(g)+2 \alpha h(g) h^{\prime}(g)+\alpha^{2} h^{\prime 2}(g)}{\oint\left[h^{2}(g)+2 \alpha h(g) h^{\prime}(g)+h^{2}(g)\right] d g}
\end{aligned}
$$

The functions $h^{2}(g),\left[h(g) h^{\prime}(g)\right]$ and $h^{\prime 2}(g)$ can be calculated independently from $\alpha$.

Once these functions are known upon a grid in Euler space, it is possible to calculate their $C$-coefficients by numerical integration, using expressions such as Eq. (27).

We now have:

$$
\begin{gathered}
h^{2}(g)=f_{s}(g)=\sum_{l} \sum_{\mu} \sum_{v} C_{s_{l}}^{\nu \mu} \dot{T}_{l}^{\mu v}(g) \\
h(g) h^{\prime}(g)=\sum_{l} \sum_{\mu} \sum_{v} C_{s_{l}}^{\mu \nu} \dot{T}_{l}^{\mu \nu} \\
h^{\prime 2}(g)=\sum_{l} \sum_{\mu} \sum_{v} C_{s_{l}}^{\prime \mu \nu} \dot{T}_{l}^{\mu \nu}
\end{gathered}
$$

The $C$-coefficients of $f_{s+1}(g)$ are (Eq. (32)):

$$
C_{s+1}^{\mu \nu}=\frac{C_{s_{l}}^{\mu \nu}+2 \alpha C_{s_{l}}^{\mu \nu}+\alpha^{2} C_{s_{l}}^{\prime \mu \nu}}{C_{s_{0}}^{11}+2 \alpha C_{s_{0}}^{\prime 11}+\alpha^{2} C_{s_{0}}^{\prime 11}}
$$

This allows the calculation of $\Delta_{s+1}$ as a function of $\alpha$. For version (a) of the method, $\Delta_{s}$ is given by Eq. (6). This leads to:

$$
\Delta_{s+1}=\sum_{l=0(2)}^{L} \sum_{\mu=1}^{M(l)} \sum_{v=1}^{N(l)} \frac{1}{2 l+1}\left(C_{s+1_{l}}^{\mu v}-C_{l}^{\mu v}\right)^{2}
$$

in which $C_{l}^{\mu v}$ are known (input data) and $C_{s+1}^{\mu v}$ are given by Eq. (36). This allows the calculation of $\Delta_{s+1}(\alpha)$ for a large number of $\alpha$ values. The value of $\alpha$ which minimizes $\Delta_{s+1}$ can now be chosen for the next estimation of $H_{l}^{\mu \nu}$. 


\section{APPENDIX 2: INITIAL ESTIMATE OF $h(g)$}

The method proposed here in order to choose an initial estimate of $h(g)$ uses the reduced O.D.F. $\tilde{f}(g)$ which is obtained from measured pole figures. In addition, a rough estimation of $\widetilde{f}(g)$ is also used. Let this rough estimation be $\widetilde{f}^{\prime}(g)$.

The following function is defined:

$$
\begin{array}{ll}
f_{0}(g)=\tilde{f}(g)+\tilde{f^{\prime}}(g) \text { when } \tilde{f}(g)+\tilde{f^{\prime}}(g) \geqslant 0 \\
f_{0}(g)=0 \quad \text { when } \tilde{f}(g)+\tilde{f}^{\prime}(g) & <0
\end{array}
$$

The initial estimate of $h(g)$ then would be

$$
h_{0}(g)=\sqrt{f_{0}(g)}
$$

The corresponding $H_{l}^{\mu \nu}$ coefficients are found by numerical integration.

\section{Rough estimate of $\tilde{\mathbf{f}}(\mathbf{g})$}

$\tilde{f}^{\prime}(g)$ is found as follows. Figure 9 gives a one-dimensional representation of the reduced texture function $\tilde{f}(g)$. A cut-off level $a_{1}$ is chosen for example at $70 \%$ of the maximum height of $\tilde{f}(g)$. The following function is not considered:

$$
\begin{array}{ll}
f_{c}(g)=\tilde{f}(g) & \text { if } \tilde{f}(g) \geqslant a_{1} \\
f_{c}(g)=0 & \text { if } \tilde{f}(g)<a_{1}
\end{array}
$$

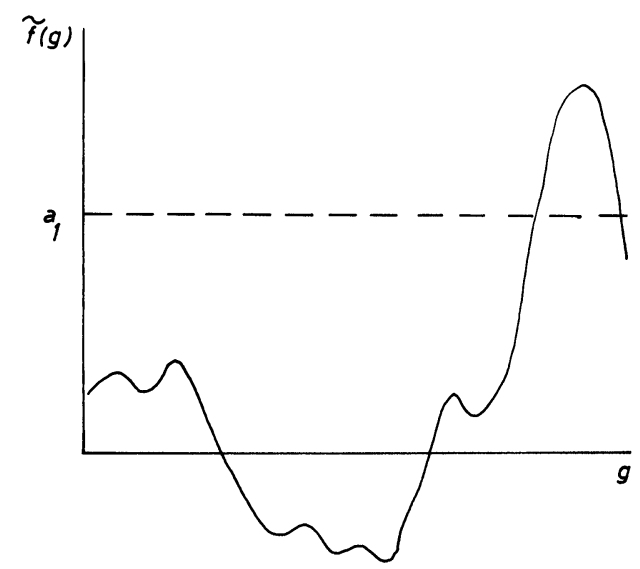

FIGURE 9 One-dimensional representation of a reduced O.D.F. and a cut-off level $a_{1}$. 
The function $f_{c}(g)$ has its own series expansion. Its odd part $\tilde{f}_{c}(g)$ is calculated by numerical integration ( $l$ up to 21$)$.

It is now hoped, that $\beta \tilde{\tilde{f}}_{c}(g)$ is an estimate (very rough indeed) of $\tilde{\tilde{f}}(g)$. The parameter $\beta$ is adjusted in such a way that the negative part of the resulting texture function is as small as possible:

$$
\int_{f^{\prime}(g)<0}\left|f^{\prime}(g)\right| d g=\text { Min }
$$

with

$$
f^{\prime}(g)=\tilde{f}(g)+\beta \tilde{\tilde{f}}(g)
$$

The minimum of Eq. (39) with respect to $\beta$ can be found by a simple algorithm which does not need much computer time, provided $f^{\prime}(g)$ and $\tilde{f}_{c}(g)$ have previously been calculated on a grid in Euler space.

In Figure 9, the function $\widetilde{f}(g)$ is now replaced by the "optimized" function $f^{\prime}(g)$. A new cut-off level $a_{2}$ is now chosen, for example at $50 \%$ of the maximum height of $f^{\prime}(g)$. The whole procedure is repeated several times. The iteration is stopped when the minimum found by Eq. (39) is larger than the previous one. The odd function $\beta \tilde{f}_{c}(g)$ corresponding to the latter is then considered to be a rough estimate of $\tilde{f}(g)$. Cut-off levels of $70 \%, 50 \%, 30 \%, 20 \%, 15 \%, 10 \%$ and $5 \%$ were used in the present work.

For most sharp textures, the resulting estimation of the odd part of the O.D.F. has been found to be good enough to reduce the ghost maxima to unsignificant levels. 